\title{
Simuliidae (Diptera, Culicomorpha) no Brasil - XII sobre o gênero Thyrsopelma Enderlein, 1934: descrição de T. jeteri Sp.N., considerações sobre o cibário e chave para as pupas
}

\begin{abstract}
Victor PY-DANIEL ${ }^{1}$, Sandra Magni DARWICH ${ }^{2}$, Lúcia Beatriz MARDINI ${ }^{3}$, Milton Norberto STRIEDER ${ }^{4}$, Sixto COSCARÓN $^{5}$

\section{RESUMO}

É acrescentado um novo caráter pupal para a espécie T. birtipupa (Lutz,1910). É evidenciada a existência de dentes no cibário da fêmea de T. guianense (Wise,1911), principal espécie envolvida na transmissão da filaria Onchocerca volvulus (Leuckart,1893) no foco da região amazônica. É proposta uma nova relação para os artículos antenais da larva de T. guianense. É descrita uma nova espécie para o gênero Thyrsopelma Enderlein,1934, T. jeteri sp.n., proveniente da bacia hidrográfica do rio Uruguai, no Estado do Rio Grande do Sul. São apresentados os parâmetros físico-químicos do criadouro de onde provem o material tipo. É apresentada uma chave para diferenciação das pupas das espécies constantes neste gênero.
\end{abstract}

\section{PALAVRAS-CHAVE}

Thyrsopelma, Thyrsopelma jeteri sp.n., taxonomia, Simuliidae, Neotropical

\section{Brazilian Simuliidae (Diptera, Culicomorpha) - XII about the genus Thyrsopelma Enderlein, 1934: description of the T. jeteri new species, considerations related with cibarium and key for the pupae}

\begin{abstract}
A new pupal character is increased for T. hirtipupa (Lutz,1910). The existence of teeth is evidenced in the female cibarium of T. guianense (Wise,1911), main species involved in the transmission of Onchocerca volvulus (Leuckart,1893) in the focus of the Amazonian area. A new relationship is proposed among the antenna articles of the T. guianense larva. A new species is described for the genus Thyrsopelma Enderlein,1934, T. jeteri sp.n., collected in Uruguay basin, State of Rio Grande do Sul. The physicochemical parameters are presented for the type locality. A key is presented for the pupal differentiation inside of Thyrsopelma.
\end{abstract}

KEYWORDS

Thyrsopelma, Thyrsopelma jeteri sp.n., taxonomy, Simuliidae, Neotropical

\footnotetext{
${ }^{1}$ Laboratório de EtnoEpidemiologia, Coordenação de Pesquisas em Ciências da Saúde, Instituto Nacional de Pesquisas da Amazônia, C.Postal 478, 69011-970, Manaus, AM, Brasil, E-mail: pydaniel@inpa.gov.br

${ }^{2}$ Centro Federal de Educação Tecnológica do Amazonas (CEFET-AM), Av. 7 de Setembro 1975, Manaus, AM, Brasil, E-mail: magnidarwich@vivax.com.br

${ }^{3}$ Centro Estadual de Vigilância em Saúde, Divisão de Vigilância Ambiental em Saúde, Secretaria da Saúde do Rio Grande do Sul, Rua Domingos Crescêncio 132, 90.650090, Porto Alegre, RS, Brasil, E-mail: lucia-mardini@saude.rs.gov.br

${ }^{4}$ Laboratório de Entomologia, Ciências da Saúde, Universidade do Vale do Rio dos Sinos, Av. Unisinos 950, 93022-000, São Leopoldo, Rio Grande do Sul, Brasil, E-mail: strieder@bios.unisinos.br

${ }^{5}$ Facultad de Ciências Naturales y Museo, Paseo del Bosque, 1900, La Plata, Argentina, E-mail: sixtoco@museo.fcnym.unlp.edu.ar
} 


\section{ACTA \\ AMAZONICA}

SIMULIIDAE (DIPTERA, CULICOMORPHA) NO BRASIL - XII SOBRE O

GÊNERO Thyrsopelma ENDERLEIN, 1934: DESCRIÇÃO DE T. Jeteri SP.N.,

CONSIDERAÇÕES SOBRE O CIBÁRIO E CHAVE PARA AS PUPAS

\section{INTRODUÇÃO}

O gênero Thyrsopelma foi descrito por Enderlein em 1934, com base em Simulium orbitale (Lutz,1910). Mais recentemente a sua validade foi questionada por pesquisadores, principalmente ingleses, que desenvolvem uma linha taxonômica para Simuliidae do tipo "alfa" \{Shelley et. al. (1997), Crosskey \& Howard (2004)\}. Uma outra linha taxonômica, centrada na filogenia, de pesquisadores com origem sulamericana, Coscarón (1987,1991), Miranda-Esquivel \& Coscarón (2001), da qual os autores deste trabalho fazem parte, consideram o mesmo válido.

Com a descrição de T. jeteri sp.n., o gênero Thyrsopelma passa a contar com sete espécies [T. scutistriata (Lutz,1909); T. birtipupa (Lutz,1910); T. orbitale (Lutz,1910); T. guianense (Wise,1911); T. itaunense (D’Andretta \& González,1964); T. perplexa (Shelley, Maia-Herzog, Luna Dias \& Couch,1989)]. Miranda-Esquivel \& Coscarón (2001), ainda tratando ao nível de subgênero, aceitam que Grenieriella (Vargas \& Nájera,1951), deva entrar na sinonímia de Trichodagmia Enderlein, 1934 e mantêm Thyrsopelma Enderlein,1934 como táxon válido, proposta a qual concordamos integralmente.

\section{GÊNERO Thyrsopelma}

\section{T. birtipupa (Lutz,1910)}

(Figs. 1 a-c)

Na re-descrição da pupa de T. birtipupa, Maia-Herzog et al. (1985) não citam a existência de tricomas cefálicos ou torácicos. Coscarón (1991) cita a existência de tricomas faciais bífidos, não diferenciando os tricomas frontais e torácicos dos espinhos que existem em grande quantidade tanto na cabeça como no tórax.

Na realidade existem longas tricomas, hialinas e finas, tanto cefálicas (frontais) como torácicas, sendo pouco evidentes porque esta espécie também apresenta grande quantidade de
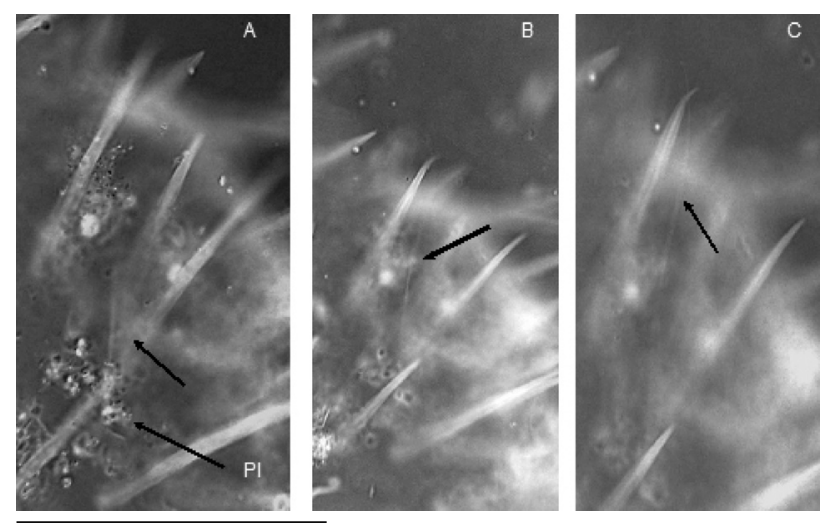

Figura 1 - Thyrsopelma birtipupa (Lutz,1910): gradação de visibilidade de uma tricoma torácica pupal, a. região basilar (PI $=$ ponto de inserção), b. região central, c. região apical. As cores das imagens foram invertidas no sentido de dar visibilidade à tricoma que é hialina. projeções cuticulares esclerotizadas, semelhantes a longas setas espatuliformes, que dificultam a visão das tricomas.

Para serem observadas é necessário procurar o lugar de inserção das mesmas, que normalmente é comum para a maioria das espécies da família Simuliidae (Figura 1a-c).

Esta espécie foi, recentemente, assinalada para a região Amazônica, na Cachoeira Santo Antonio, rio Jarí, Estado do Amapá (Hamada et al.,2003; Bastos et al., 2004).

Material examinado: INPA n ${ }^{\circ} 6054$ (3 pupas / 5 larvas), Localidade Cachoeirinha, $30 \mathrm{Km}$ após a Cidade Paraíso do Norte de Goiás (sentido Goiás = > Pará), Br-153, 08.i.1976, cols. V.PyDaniel \& L.H. Rapp.

\section{T. guianense (Wise, 1911)}

\section{(Figs. 2 a-b)}

Esta espécie possui uma ampla distribuição geográfica na América do Sul que abrange desde a região sudeste do Brasil até a Venezuela, Guiana, Suriname e o Departamento francês também denominado de Guiana. No foco oncocercótico da área Yanomami/Ye'kuana, que abrange o Sul-Sudeste da Venezuela (Estados do Amazonas e Bolívar) e Norte-Noroeste do Brasil (Estados do Amazonas e Roraima), ela é assinalada como a principal vetora da filária Onchocerca volvulus (Leuckart,1893) (Py-Daniel,1997).

\section{Considerações sobre o cibário}

Shelley et al. (1997) e Shelley et al. (2000) examinando exemplares, tanto para a região centro-oeste do Brasil como para a própria região focal amazônica, indicam que as fêmeas desta espécie não possuem dentes no cibário.

Uma das características assinaladas como importante para a eficiência transmissiva de uma espécie é a ausência de dentes no cibário da fêmea. A presença de dentes indicaria a maior possibilidade de rompimento das microfilárias quando da ingestão do sangue, agregando assim um fator de dificuldade na continuidade do ciclo do parasito.

Entre as espécies assinaladas para o foco da Amazônia, $T$. guianense é reconhecida como a mais eficiente (Py-Daniel et al., 2000).

Como pode ser observado na Figura 2a,b e também na figura 56 de Shelley et al.(1997), os cibários das fêmeas de guianense possuem grande número de dentes com o ápice rombo e normalmente mais largos que longos, não sendo muito destacados do tegumento do cibário. Portanto, a afirmação de que o cibário da fêmea de T. guianense não possui dentes é um erro.

É óbvio que dentes agudos, se comparados com dentes rombos, propiciam uma maior barreira na ingestão das microfilárias, mas dentes rombos, com seus ápices irregulares, também constituem um impedimento. Assim, com base nesta evidência, certamente existem outras justificativas para a relação de eficiência de T. guianense na transmissão de $O$. volvulus, 


\section{ACTA AMAZONICA}

SIMULIIDAE (DIPTERA, CULICOMORPHA) NO BRASIL - XII SOBRE O

GÊNERO Thyrsopelma ENDERLEIN, 1934: DESCRIÇÃO DE T. Jeteri SP.N., CONSIDERAÇÕES SOBRE O CIBÁRIO E CHAVE PARA AS PUPAS além de ser uma espécie cujas fêmeas possuem um tamanho grande, o que possibilita maior carga parasitária.

\section{Chave para as quatro espécies envolvidas na transmissão (segundo Py- Daniel, 1997) de O. volvulus na Região Amazônica, quanto à forma e locais de inserção dos dentes do cibário das fêmeas.}

1. Cibário plano (sem reentrância central) 2

- Cibário com reentrância central . 3

2. Cibário sem dentes ... Notolepria exiguua (Roubaud,1906)

- . Cibário apenas com dentes rombos ......... Thyrsopelma guianense (Wise,1911)

3. Reentrância do cibário pouco profunda e com dentículos do tipo rombo ........ Psaroniocompsa incrustata (Lutz, 1910)

- . Reentrância do cibário profunda e sem dentículos do tipo rombo ...... Cerqueirellum oyapockense (Floch \& Abonnenc, 1946)
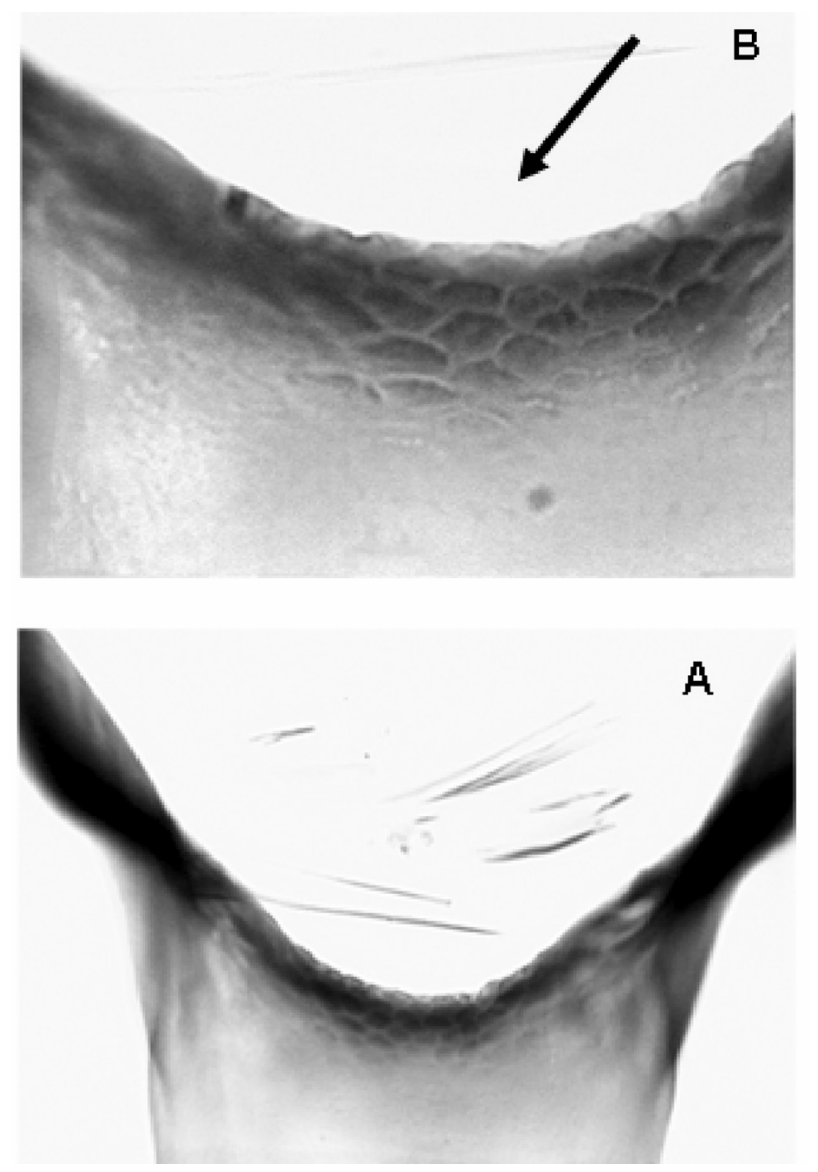

Figura 2 - Thyrsopelma guianense (Wise,1911): a. região apical do cibário; b. pormenor dos dentes rombos do cibário.
Shelley et al. (1997) apresentam, para T. guianense, uma proporção dos segmentos antenais da larva de 35:10:22. Esta proporção, para um grande número de larvas examinadas, não representa a realidade que foi encontrada aqui, assim é proposto substituir a proporção apresentada por eles pela seguinte: I:II:III = 1:1,44-1,97:1-1,17. Esta relação é encontrada em todas as outras espécies de Thyrsopelma (faltando examinar $T$. perplexa, cuja a larva ainda é desconhecida), e pode ser expressa por $=$ II $>$ I $\geqslant$ III.

Material examinado de T. guianense: INPA n 5861 (muitas larvas e pupas), Localidade: Rio Oiapoque, Cachoeira de Maripa (vários saltos, incluso o Cafésoca), divisa binacional entre Brasil e França (Departamento da Guiana), 24-25.ix.1981, col. V. PyDaniel.

\section{Thyrsopelma jeteri sp.n.}

(Figs. 3 a-e, 4 a-h, 5 a-f, 6 a-f, 7 a-f, 8 a-f, 9 a-h)

FÊMEA - Forma geral conforme a figura $3 a$. Fronte alta com Triângulo Fronto Ocular profundo (Fig. 3c). Antena conforme a figura 3b. Maxila com 26-27 dentes (11-15 externos / 12-13 internos / 1 apical). Mandíbula com apenas 27 dentes internos (sem externos). Calcipala e pedisulco presentes (Fig. 3d). Unhas das garras tarsais do terceiro par de pernas sem dente acessório (Fig. 3e).

MACHO - Forma geral conforme a figura 4 a. Antena e palpo maxilar conforme as figuras 4b-c. Distímero mais longo que o Basímero (Fig. 4f). Distímero apresentando-se mais afilado no ápice que na base (Fig. 4f) e com 1 espinho apical (grande, achatado) e outro (que pode estar ausente) menor e mais afilado (Fig. 4g-h). Furcasterno conforme a figura 4d. Com calcipala e pedisulco presentes (Fig. 4e).

PUPA - Coloração geral (no álcool) castanho clara. Casulo em forma de sapato, não cobrindo as brânquias, com textura lisa, sem projeção antero-dorsal (Fig. 5a). Comprimento dorsal do casulo: $2,18-2,5 \mathrm{~mm}(\mathrm{n}=5)$. Comprimento ventral do casulo: 2,59-2,96 mm ( $\mathrm{n}=4)$, Porção anterior do casulo: 0,025-0,5 mm $(\mathrm{n}=4)$. Porção posterior do casulo: $2,25-2,5 \mathrm{~mm}(\mathrm{n}=4)$. Fórmula branquial $(\mathrm{n}=12)=1[(1)+1[(1)+1(2)]]+1[1[1(2)+1(2)]+$ $1(2)]+1[(2)]+1[1(1)+1(2)]$, com um total de ramos terminais igual a 15. Superfície dos filamentos branquiais coberta com pequenos tubérculos espiniformes com o ápice arredondado (Fig. 5e). Ápice dos filamentos branquiais esclerotizado e afinado, sendo os ramos primários muito mais largos que os terminais (Fig. 5b-d). Forma geral das brânquias do tipo cônico. Brânquias com 0,61-0,8 mm de comprimento. Fronto-clípeo variando desde sem tubérculos até com grande quantidade, sendo os mesmos pequenos, arredondados (Fig. 5f). Frontoclípeo sem expansões, com tricomas frontais presentes, curtas (reduzidas), com ápice simples, em número de 1+1 (Fig. 6c); com tricomais faciais presentes, curtas (reduzidas), com oápice simples, em número de $1+1$. As tricomas faciais maiores que as tricomas frontais (FA>FR). Estojos antenais lisos e sem espinhos. Tricomas epicrâniais, genais e oculares ausentes. Tórax sem ou com tubérculos (a região anterior apresenta 


\section{ACTA AMAZONICA}

SIMULIIDAE (DIPTERA, CULICOMORPHA) NO BRASIL - XII SOBRE O

GÊNERO Thyrsopelma ENDERLEIN, 1934: DESCRIÇÃO DE T. Jeteri SP.N.,

CONSIDERAÇÕES SOBRE O CIBÁRIO E CHAVE PARA AS PUPAS
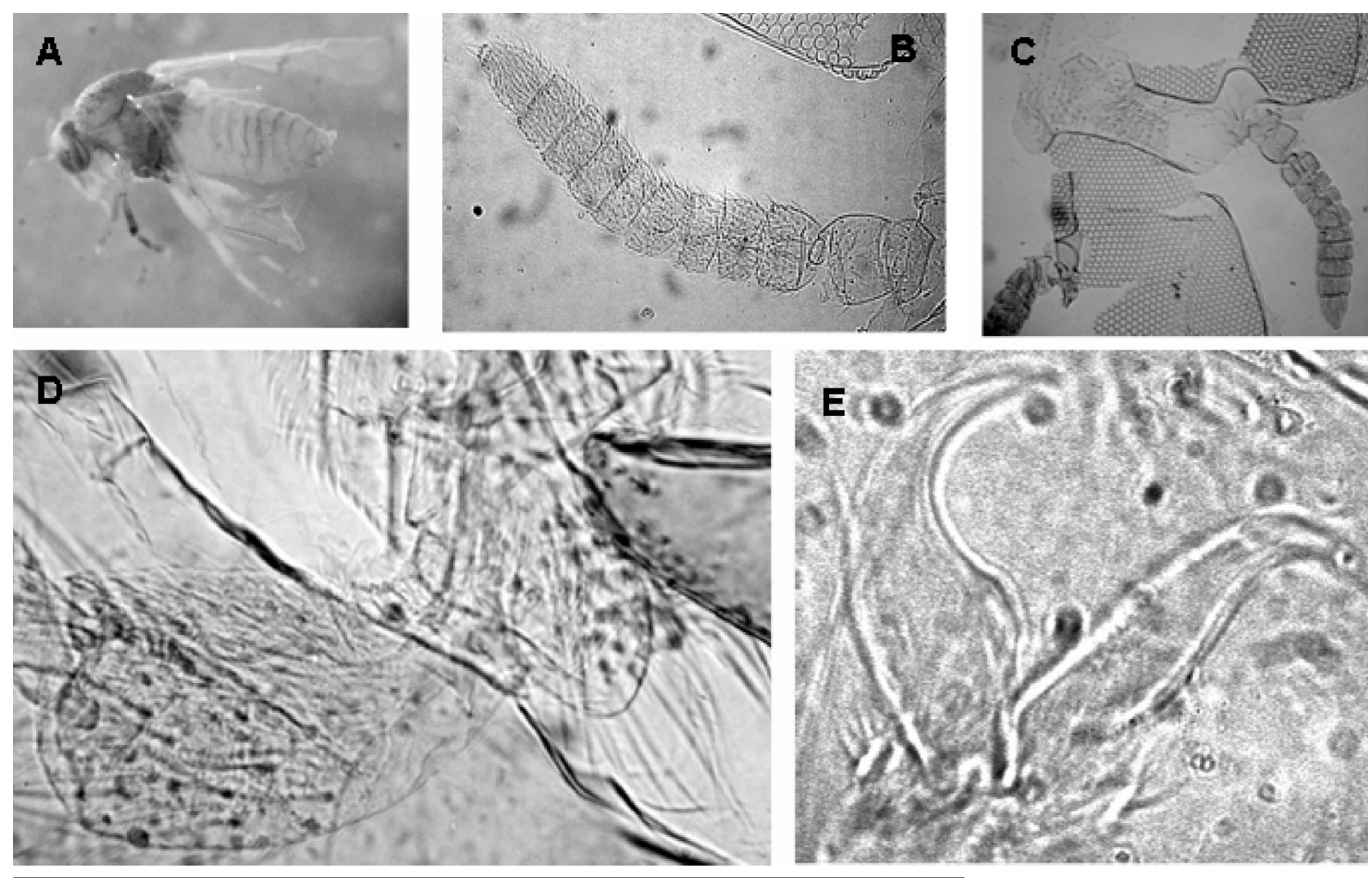

Figura 3 - Thyrsopelma jeteri sp.n. - fêmea: a. aspecto geral; b. antena; c. fronte, antena e ângulo frontal; d. calcipala e pedisulco; e. unhas das garras tarsais do terceiro par de pernas.

esporadicamente tubérculos, as regióes posterior e lateral com grande quantidade de tubérculos, podendo estar presentes apenas na região posterior, em grande número, pequenos $\mathrm{e}$ subtriangulares) arredondados, com tamanho médio. Tricomas torácicas: $5+5$ centro-dorsais (a mais ventral longa e o restante curta), $1+1$ supra-laterais (curtas), $3+3$ laterais (médias e/ou curtas) (Fig. 6d), sendo todas com o ápice simples, espiniformes (exceto as $1+1$ centro-dorsais mais ventrais e as 1+1 laterais superiores que são filiformes). Tricomas torácicas laterais apresentando a distribuição do tamanho: S (Superior) > I (Inferior) > M (Mediana). Pteroteca (fig. 5b) coberta com tubérculos arredondados pequenos. Ausência de manchas torácicas. Abdome totalmente membranoso, sem carenas (Fig. 6A-B). Tergito abdominal I: com $1+1$ setas longas, laterais, sem ganchos, sem ou com dentículos, do tipo pequeno, dispostos em $1+1$ áreas anteriores. Tergito abdominal II: com $1+1$ setas, simples, longas, medianas e $1+1$ setas curtas / 4+4 ganchos também curtos, sem dentículos anteriores. Tergitos abdominais III-IV: com $2+2$ setas, simples, medianas, látero-externas e $4+4$ ganchos também simples e posteriores (Fig. 6f), com 1+1 áreas antero-laterais apresentando dentículos do tipo pequeno. Tergito abdominal V: $\operatorname{com} 5+5$ ou $6+6$ (sendo $1+1$ muito reduzidas, laterais) setas, simples, posteriores, curtas e com dentículos anteriores do tipo pequeno. Tergito abdominal VI: com $2+2$ setas, simples, curtas, posteriores e com dentículos anteriores do tipo pequeno. Tergito abdominal VII: com $2+2$ setas, com dentículos anteriores do tipo pequeno. Tergito abdominal VIII: com $2+2$ setas, simples, posteriores, curtas, com dentículos anteriores do tipo pequeno. Tergito abdominal IX: sem setas, com dentículos anteriores do tipo pequeno. Ganchos terminais presentes $\mathrm{e}$ extremamente reduzidos. Extremidade terminal do abdome afilada. Látero-tergitos (região pleural circundante aos espiráculos) ausentes. Áreas esclerotizadas nas membranas inter-segmentares ausentes. $3+3$ setas pleurais (circundantes aos espiráculos), simples. Micro-setas inter-segmentares presentes. Segmentos abdominais esternais III-IV: $1+1$ áreas anteriores com dentículos em forma de pente, com $2+2$ setas, simples, sem ganchos. Segmento abdominal esternal V: com dentículos em forma de pente, na região anterior, $\operatorname{com} 3+3$ setas, simples, com área membranosa estriada medianalongitudinal apenas no terço posterior, sem ganchos. Segmento abdominal esternal VI: dentículos em forma de pente presentes em $1+1$ áreas anteriores, com $2+2$ setas, simples externas e $2+2$ ganchos bífidos (pode aparecer trífido; sendo que o gancho interno pode ser setiforme e apresentando apenas a base - área de inserção - esclerotizada), área membranosa estriada mediana-longitudinal presente e completa. Segmento abdominal esternal VII (Fig. 6e): com dentículos em forma de pente, frontais ao gancho interno, com $3+3$ setas, simples, pequenas e $2+2$ ganchos, maiores, 


\section{ACTA AMAZONICA}
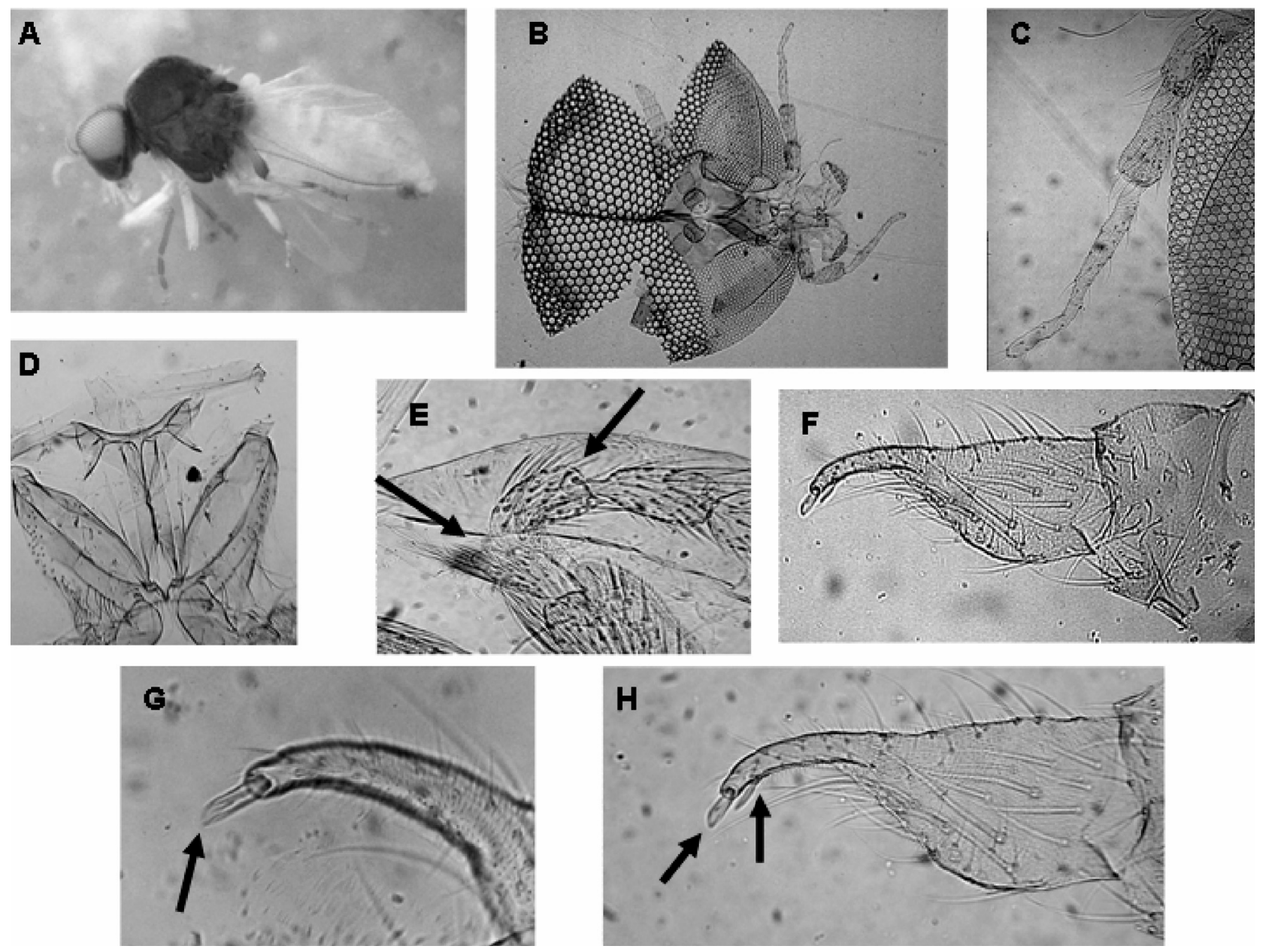

Figura 4 - Thyrsopelma jeteri sp.n. - macho: a. aspecto geral; b. antena, palpo e fronte; c. palpo maxilar; d. furcasterno; e. calcipala e pedisulco; f. basímero e distímero; g-h. variações do número de espinhos no ápice do distímero.

bífidos, área membranosa estriada mediana-longitudinal presente e completa. Segmento abdominal esternal VIII: dentículos em forma de pente frontais ao gancho interno, com 2+2 ganchos bífidos (ou sem ganchos), com setas (ou sem setas), área membranosa estriada mediana-longitudinal presente e completa.

LARVA - Coloração geral, ao vivo, cinza-esverdeada, no álcool amarelo-esbranquiçado. Comprimento do corpo variando entre 5,25-5,63 mm. Contorno do corpo roliço e com a parte posterior mais alargada (Fig. 7a-c)). Histoblasto branquial, curto e arredondado (Figs. 7d, 8b) Cabeça menos larga (Fig. 7e) que o tórax. Histoblastos posteriores falciformes (Fig. $7 \mathrm{f}$ ). Cutícula abdominal com grande quantidade de escamas do tipo subpetalóides (Fig. 8e,f), sendo que na região posteriordorsal apresentam-se em maior número, maiores e mais esclerotizadas. Sem projeções e manchas cuticulares dorsais. Um tubérculo posterior ventral, subtriangular, aparecendo mais nitidamente quando o abdome está totalmente distendido (semelhante ao encontrado em Stegopterna e Cnephia.).
Tubérculos posteriores laterais ausentes. Brânquias anais múltiplas, com três lóbulos principais, apresentando cada um, divertículos que variam em número de 10-11. Máxima largura da cápsula cefálica com $0,70 \mathrm{~mm}$. Leques cefálicos presentes. Antena (Fig. 8a) mais longa que a haste do leque cefálico. Número de segmentos antenais igual a 3; o terceiro segmento antenal é pouco mais escuro que os segmentos I-II; segmentos antenais sem estriações transversais ou longitudinais; relações entre os segmentos antenais (III $<$ II II $>$ I; III $>$ I); proporção dos segmentos antenais: I:II:III $=1: 1,73-1,86: 1,19-1,54$. Manchas no apódema fracamente positivas. Apódema sem desenhos (Fig. 7e); não visualizadas setas no apódema; apódema com a região central menos larga que a apical. Escleritos cervicais grandes, alongados e livres na membrana. Número de raios do leque cefálico variando entre 51-56; setas do terço apical dos raios do leque cefálico, filiformes; manchas dos raios dos leques cefálicos ausentes; fibrilas dos leques cefálicos do tipo "padrão"; região basilar dos raios dos leques cefálicos nitidamente mais alargada (Fig. 8c), mais curta que a 


\section{ACTA AMAZONICA}
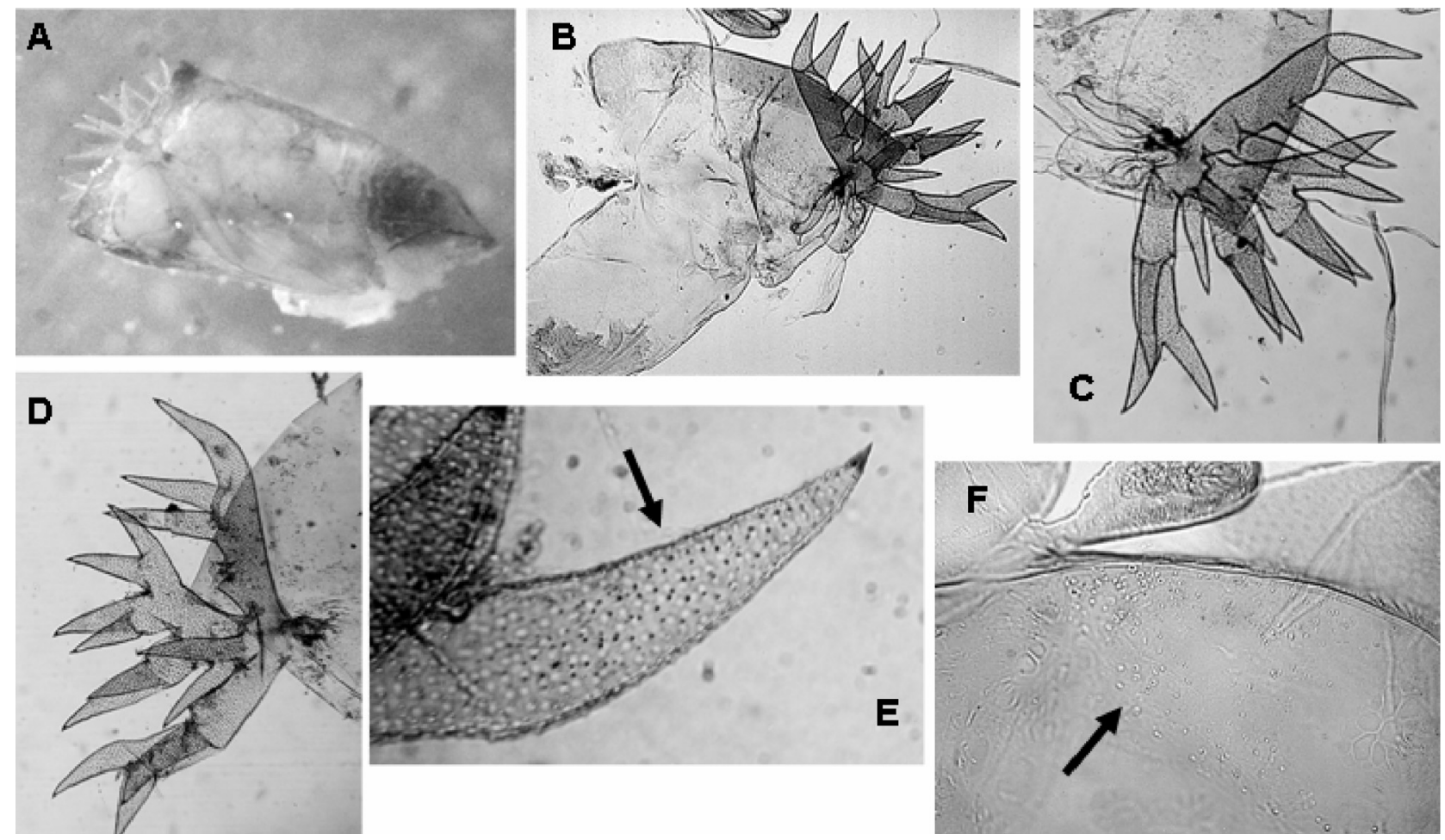

Figura 5 - Thyrsopelma jeteri sp.n. - pupa: a. aspecto geral da pupa evidenciando as proporções entre as brânquias e o casulo; b. pteroteca, tórax e brânquia; c-d. variações das brânquias torácicas; e. ápice de um filamento terminal branquial evidenciando os tubérculos espiniformes; f. vista parcial do fronto-clípeo pupal evidenciando a forma e o pequeno número de tubérculos.

região apical (1:4,62); ápice da região basilar dos leques cefálicos sem espinho. Raios petaliformes dos leques cefálicos em número de 11. Serrilhas laterais do hipostômio ausentes; disposição dos dentes do tipo simples (com os dentes pontas/ central e intermediários não diferenciados em tamanho), mas o central e os 1+1 intermediários internos são mais estreitos, sendo que todos dentes apresentam alargamento basilar (Fig. 9e-f); dentes hipostomiais pontas com o ápice direcionado para frente; dentes laterais do hipostômio, ausentes ou com 1 (que apareceu em apenas um dos lados) (Fig. 9g). Sem projeção laminar hipostomial; número total dos dentes hipostomiais = 9 (mas podem ser 10 quando aparece um dente externo). Proporção entre Fenda Gular : Ponte Pré-Gular : Hipostômio = 1: 0,43:0,54 (Fig. 9d). Proporção entre Ponte PréGular:Hipostômio $=1: 1,25$. Número de fileiras de setas hipostomiais $=1$ (com 5-7 setas). Fenda Gular profunda (maior que a PPG) e com forma variando entre circular e sub-mitral. Dentes externos da mandíbula em número de dois; dentes pré-apicais e internos indiferenciados quanto à inserção e forma (apenas sendo os pré-apicais maiores) (Fig. 9b); dentes préapicais +internos em número de 8-9; I dente pré-apical maior que o III pré-apical; dente apical maior que o III pré-apical; tubérculos látero-frontais no dente apical ausentes; setas supramarginais da mandíbula ausentes; setas posteriores aos dentes internos, ausentes; dentes marginais, dispostos em apenas um conjunto de 2 , sendo o anterior maior que o posterior;
PLM presente (Fig. 9c), com inserção no centro-anterior do RBI (reforço basilar inferior), com o ápice direcionado para a região frontal da mandíbula, simples, reto; área supra escova distal adoral apresentando-se hialina na região de inserção das setas da escova. Esclerito labral sub-trapezoidal com setas anteriores finas, e com $3+3$ dentes anteriores direcionados para frente (Fig. 9a). Esclerito lateral do pseudópodo com setas apicais dispostas em 7 grupos (Fig. 8d). Setas peri-anais ausentes. Esclerito anal simples, em forma de X (Fig. 9h), sem escleritos acessórios e sem estruturas longitudinais de rigidez, tendo os braços posteriores mais longos que os anteriores. Número fileiras de ganchos do disco anal = 130-164; número de ganchos por fileira = 14-19. Áreas esclerotizadas, do láteroesclerito anal, mais alargadas anteriormente.

Etimologia: O nome desta espécie é uma homenagem ao Dr. Jeter Bertoletti, na época Diretor do Museu de Ciências Naturais da PUC-RS, Porto Alegre, Rio Grande do Sul, que deu o suporte necessário para o desenvolvimento das excursões que possibilitaram as primeiras coletas na bacia hidrográfica do rio Uruguai.

\section{Caracterização ambiental dos criadouros}

As primeiras coletas dos imaturos de T. jeteri sp.n. foram obtidas por meio de um Projeto de Levantamento Faunístico (Insetos de interesse médico) nas áreas de influência das 


\section{ACTA AMAZONICA}

SIMULIIDAE (DIPTERA, CULICOMORPHA) NO BRASIL - XII SOBRE O

GÊNERO Thyrsopelma ENDERLEIN, 1934: DESCRIÇÃO DE T. Jeteri SP.N., CONSIDERAÇÕES SOBRE O CIBÁRIO E CHAVE PARA AS PUPAS
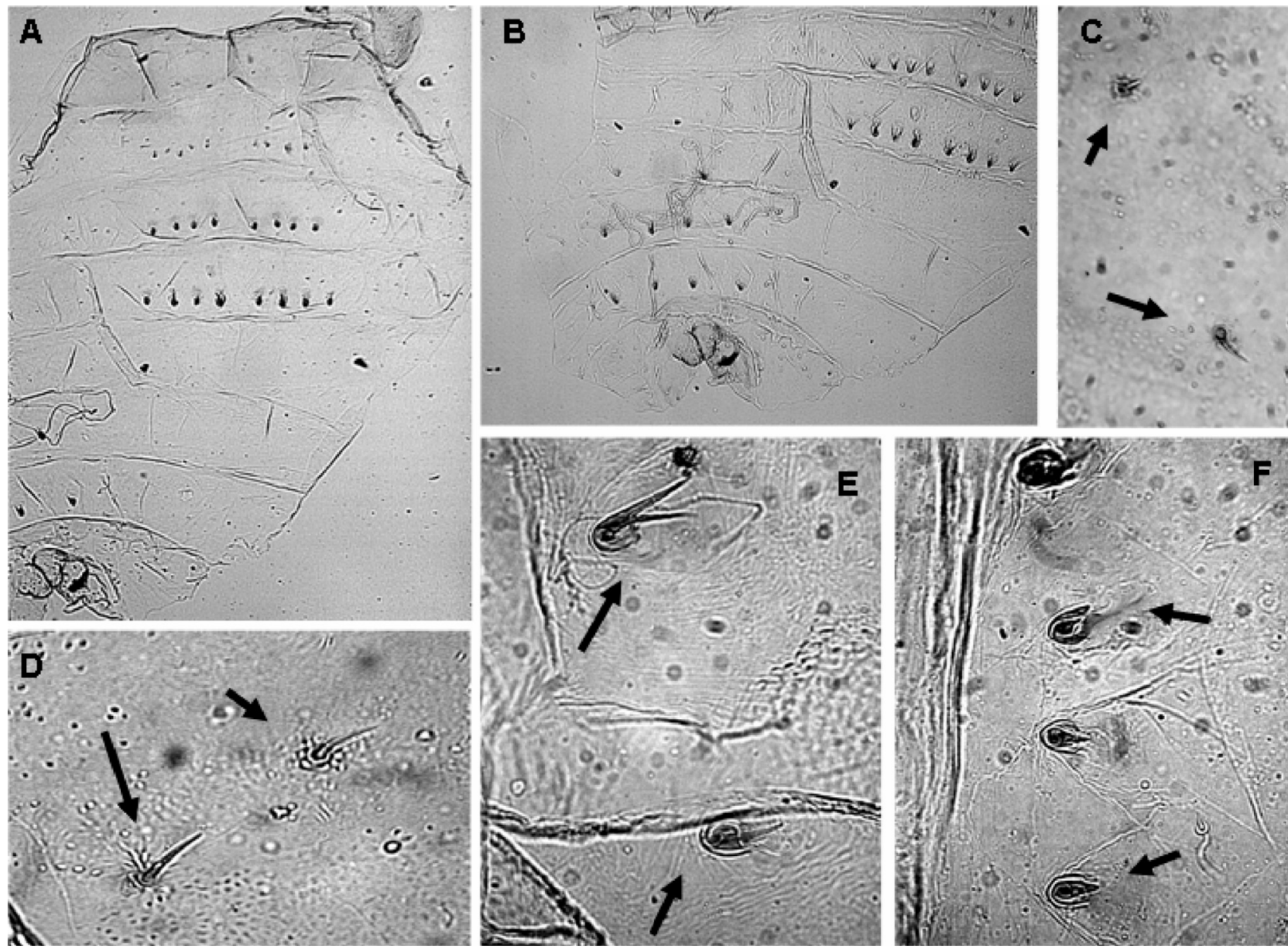

Figura 6 - Thyrsopelma jeteri sp.n. - pupa: a. tergitos abdominais; b. tergitos e esternitos abdominais; c. tricomas frontais da cabeça; d. tricomas centro dorsais do tórax; e. ganchos do esternito VII; f. ganchos do tergito IV.
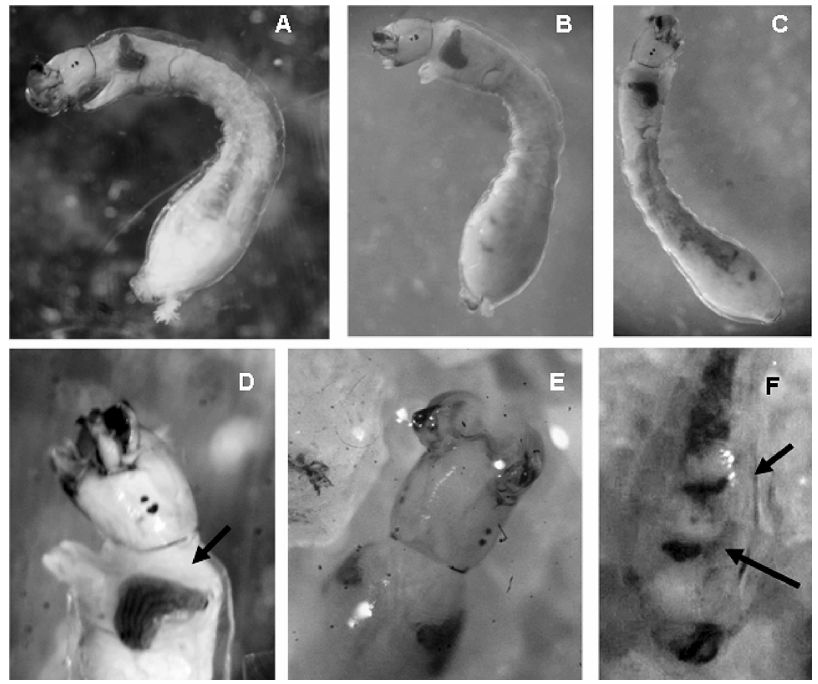

Figura 7 - Thyrsopelma jeteri sp.n. - larva: a-b-c. diferentes aspectos da conformação geral da larva; d. vista lateral evidenciando os histoblastos branquiais; e. vista dorsal da cabeça; f. histoblastos posteriores falciformes.
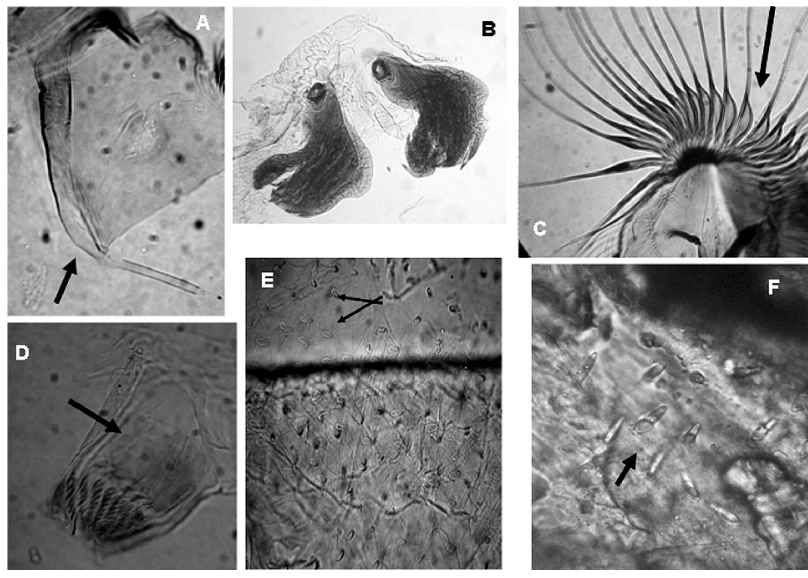

Figura 8 - Thyrsopelma jeteri sp.n. - larva: a. antena; b. histoblastos branquiais; c. leque cefálico evidenciando o alargamento da região basilar; d. esclerito lateral do pseudópodo; e-f. variações das setas espatuliformes do tegumento abdominal; 


\section{ACTA AMAZONICA}

SIMULIIDAE (DIPTERA, CULICOMORPHA) NO BRASIL - XII SOBRE O

GÊNERO Thyrsopelma ENDERLEIN, 1934: DESCRIÇÃO DE T. Jeteri SP.N.,

CONSIDERAÇÕES SOBRE O CIBÁRIO E CHAVE PARA AS PUPAS
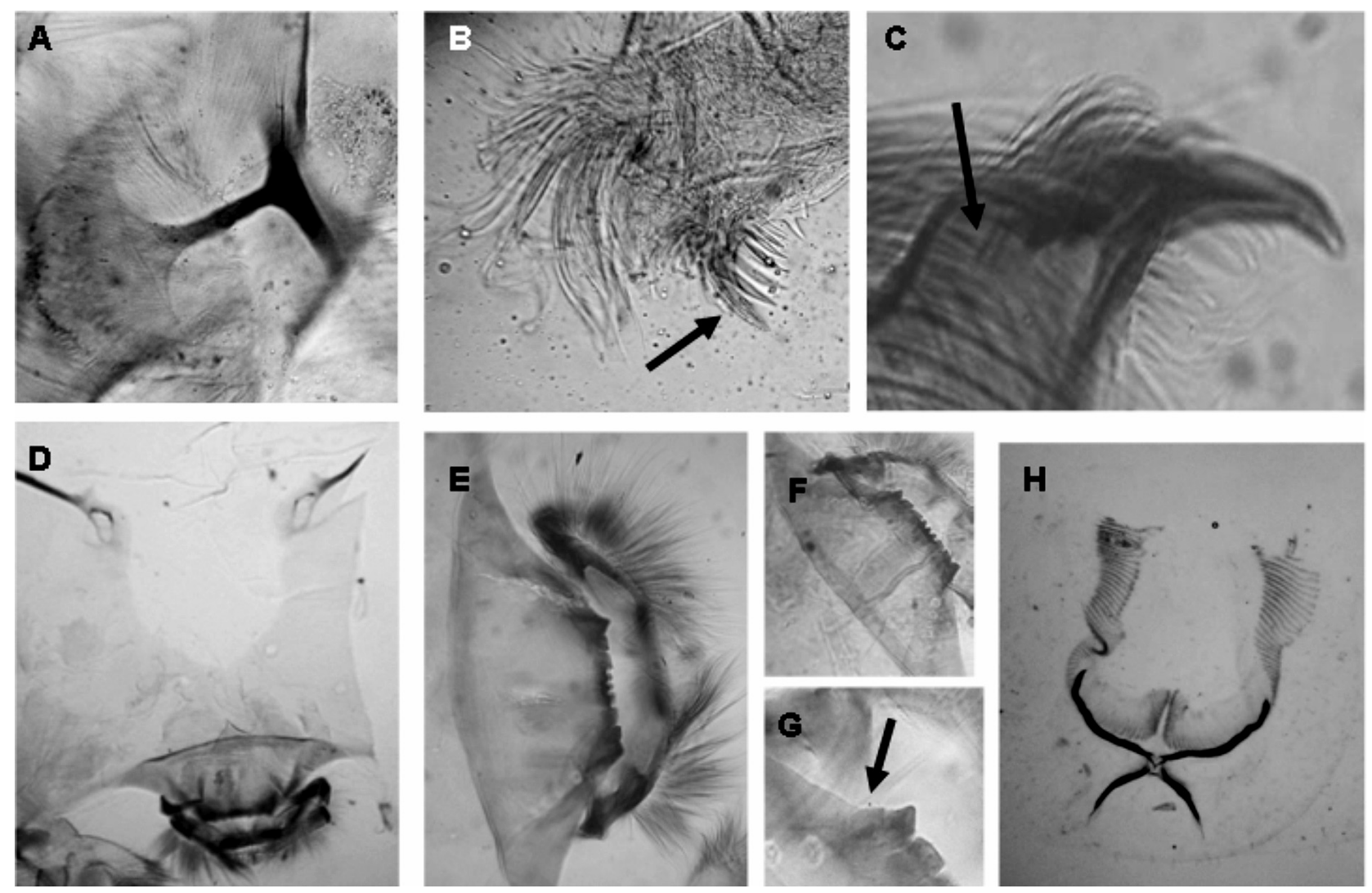

Figura 9 - Thyrsopelma jeteri sp.n. - larva: a. esclerito labral; b. ápice da mandíbula evidenciando os dentes; c. ápice da mandíbula evidenciando a base do PLM; d. hipostômio, ponte pré-gular, fenda gular; e-f. variações dos dentes do hipostômio; g. pormenor do hipostômio evidenciando a presença um dente lateral; h. Esclerito anal com disco anal e ganchos.

barragens de Itá (Santa Catarina) e Machadinho (Rio Grande do Sul). Ambas as áreas fazem parte da bacia do rio Uruguai. Estas áreas estão situadas na zona fisiográfica do Planalto sobre capeamento basalto-arenítico, sendo que o rio Uruguai (que passa a ter este nome após a confluência do rio do Peixe) é formado pela união dos rios Pelotas e Canoas.

Originalmente, ao longo do rio desenvolvia-se densa vegetação formada de araucária, cedro, louro, grapipunha, angico, açoita-cavalo e outras, entremeadas de zonas de campo. Estas matas de madeira nobre já não se encontram mais no seu estado natural, pois a intensa colonização da área fez com que as mesmas dessem lugar às lavouras de milho, feijão, soja, trigo, fumo, etc.

Os cursos d'água nos locais dos criadouros (Fig. 10) de $T$. jeteri sp.n. apresentavam características comuns como: fundo rochoso, água cristalina, margens com vegetação rarefeita, sendo os aspectos físico-químicos das águas da localidade-tipo, apresentados na Tabela 1 .

Os imaturos de T. jeteri sp.n. foram coletados em quatro localidades no Rio Grande do Sul: rio Ligeiro, Município de Maximiliano de Almeida, próximo à ponte da RS-126, após receber o rio Apuaê Mirim; rio Ligeiro, após receber o rio

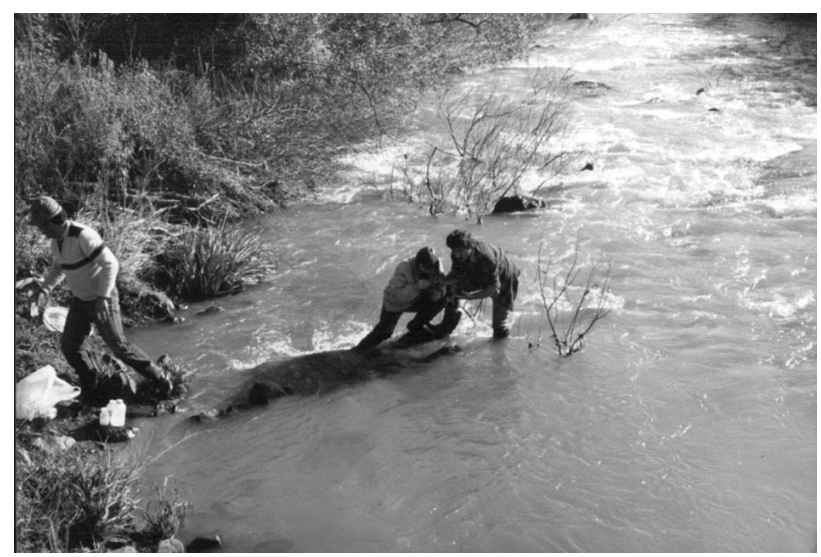

Figura 10 - Arroio Marmeleiro, município de Barracão, RS, localidade tipo de Thyrsopelma jeteri sp.n.

Piracucê, na altura de São João da Urtiga a 15,5 km da RS-126, no município de Paim Filho; rio Forquilha, Município de Maximiliano de Almeida, numa extensão de $3 \mathrm{~km}$ da ponte que liga este município até o município de Machadinho; arroio Marmeleiro, Município de Barracão, na região de Espigão Alto. 


\section{ACTA \\ AMAZONICA}

SIMULIIDAE (DIPTERA, CULICOMORPHA) NO BRASIL - XII SOBRE O

GÊNERO Thyrsopelma ENDERLEIN, 1934: DESCRIÇÃO DE T. Jeteri SP.N., CONSIDERAÇÕES SOBRE O CIBÁRIO E CHAVE PARA AS PUPAS
Tabela 1 - Parâmetros físico-químicos do arroio Marmeleiro, município de Barracão, RS, localidade tipo de Thyrsopelma jeteri sp.n., obtidos em 02.ix.1988,

\begin{tabular}{ll}
\hline \hline Disco de Secchi & $0,60 \mathrm{~m}$ \\
Coeficiente de extinção & $2,83 \mathrm{~m}-1$ \\
Penetração de luz a $1 \%$ & $1,63 \mathrm{~m}$ \\
Temperatura da água & $11,0 \mathrm{oC}$ \\
Cor & $117,33 \mathrm{mg} \mathrm{Pt} / \mathrm{l}$ \\
Potencial hidrogeniônico (pH) & 6,08 \\
Condutividade elétrica & $33,0 \mathrm{uS} 25 / \mathrm{cm}$ \\
$\mathrm{N}-\mathrm{NH} 4+$ (amônia) & $2 \mathrm{ug} / \mathrm{l}$ \\
$\mathrm{N}-\mathrm{NO}$ - (nitrito) & $4 \mathrm{ug} / \mathrm{l}$ \\
$\mathrm{N}-\mathrm{NO} 3$ - (nitrito) & $607 \mathrm{ug} / \mathrm{l}$ \\
Cloretos (Cl-) & $1,06 \mathrm{mg} / \mathrm{l}$ \\
P-PO4 3- (fosfatos) & $0,011 \mathrm{mg} / \mathrm{l}$ \\
Ca 2+ & $2,14 \mathrm{mg} / \mathrm{l}$ \\
Mg 2+ & $3,02 \mathrm{mg} / \mathrm{l}$ \\
Dureza & $3,57 \mathrm{mg} \mathrm{CaCo3/l}$ \\
Fe total & $0,706 \mathrm{mg} / \mathrm{l}$ \\
Fe dissolvido & $0,364 \mathrm{mg} / \mathrm{l}$ \\
Fe particulado & $0,342 \mathrm{mg} / \mathrm{l}$ \\
Si-Si(OH)4 & $6,138 \mathrm{mg} / \mathrm{l}$ \\
Alcalinidade & $22,88 \mathrm{mg} \mathrm{HCO3-/l}$ \\
DQO & $18,69 \mathrm{mg} \mathrm{material}$ \\
\hline \hline
\end{tabular}

\section{DISCUSSÃO}

Thyrsopelma jeteri sp.n. é separada de todas as outras espécies, dentro do gênero, pela pupa apresentar um número de filamentos branquiais terminais igual a 15 , sendo os filamentos primários muito mais largos que os filamentos apicais. Quanto ao tamanho ventral do casulo pupal, se aproxima de T. guianense (T. jeteri sp.n. $=2,59-2,90 \mathrm{~mm} ; T$. guianense $=2,2-3,6 ;$. . orbitale $=3,1-3,7 ;$ T. scutistriata $=4,0$ 4,5; T. itaunense $=4,5-4,9 ;$ T. hirtipupa $=4,0-5,0)$. Quanto ao número de fileiras de ganchos do disco anal da larva, existem dois grupos de espécies [as que variam entre 130-166: T. jeteri sp.n. (130-164), T. itaunense (133), T. orbitale (160-166) e T. guianense (143-151); e as que variam com número acima de 200: T. hirtipupa (220) e T. scutistriata (280-300)]. Quanto aos ganchos por fileiras do disco anal T. jeteri sp.n. (14-19) é uma das que apresenta menor número [T. itaunense (17-22), $T$. birtipupa (28-31), T. orbitale (25-30), T. guianense (21-28), T. scutistriata (28-35)]. Quanto ao número dos divertículos do lóbulo anal T. jeteri (10-11) se aproxima mais de T. birtipupa (12-14), T. orbitale (10-15) e T. guianense (10-12) do que das outras espécies [T. itaunense (19-22), T. scutistriata (30)]. Em relação ao comprimento das larvas de último estádio, T. jeteri sp.n. (5,25-5,63 mm) é a menor, e mais próxima de T. guianense
(3,9-6,0), sendo as outras espécies maiores [T. orbitale $(6,7), T$. itaunense (6,8), T. hirtipupa (7,3-8,8), T. scutistriata $(10-10,5)]$. Todas as espécies, quanto à proporção dos segmentos antenais larvais I:II:III apresentam a relação II >III $\geqslant$ I e um número de raios dos leques cefálicos dentro do intervalo de 45-60.

\section{MATERIAL EXAMINADO}

Material Tipo-Thyrsopelma jeteri sp.n. - Brasil, Rio Grande do Sul, Município de Barracão, localidade de Espigão Alto, Arroio Marmeleiro, 02.ix.1988, INPA n ${ }^{\circ} 6284$ (1 exúvia pupal em álcool / 2 exúvias pupais em lâmina/ 6 pupas (Parátipos)/ 1 macho retirado de pupa e respectiva exúvia pupal em lâmina (Parátipo)/ 1 fêmea retirada de pupa e respectiva exúvia pupal em lâmina (Holótipo) / 6 larvas de ultimo estádio (Parátipos) / 6 larvas em estádios diferentes), Victor Py-Daniel, Sandra Darwich, Lúcia Mardini, Ulysses C. Barbosa. O material tipo está depositado na Coleção de Simuliidae do Instituto Nacional de Pesquisas da Amazônia (INPA).

Material Adicional - Thyrsopelma jeteri sp.n. - Brasil, Rio Grande do Sul, Município de Maximiliano de Almeida, Rio Ligeiro, Pedral, 04.ix.1988, cols. Victor Py-Daniel, Sandra Darwich, Lúcia Mardini, Ulysses C. Barbosa, INPA n ${ }^{\circ} 6278$ (3 pupas, 1 larva, 1 fêmea, em álcool). São João da Urtiga, Rio Ligeiro, 03.ix.1989, cols. Victor Py-Daniel, Sandra Darwich, Lúcia Mardini, Ulysses C. Barbosa, INPA n ${ }^{\circ} 6279$ ( 3 pupas, 1 exúvia pupal, em álcool), INPA n ${ }^{\circ} 6280$ (4 larvas de último estádio em álcool e 4 em lâminas, 1 pupa no álcool). Município de Maximiliano de Almeida, Rio Forquilha, cachoeira, 04.ix.1988, cols. Victor Py-Daniel, Sandra Darwich, Lúcia Mardini, Ulysses C. Barbosa, INPA n ${ }^{\circ} 6281$ (4 pupas). Município de Barracão, localidade de Espigão Alto, Arroio Marmeleiro, 02.ix.1988, cols. Victor Py-Daniel, Sandra Darwich, Lúcia Mardini, Ulysses C. Barbosa, INPA $n^{\circ} 6283$ ( 2 pupas, 1 larva, 1 macho); INPA ${ }^{\circ}$ 6285, 03.ix.1989 (1 pupa), Victor Py-Daniel, Sandra Darwich, Lúcia Mardini, Ulysses C. Barbosa; UNISINOS nº 834, 26.xi.1996 (19 pupas / 5 exúvias pupais / 11 larvas de ultimo estádio / 1 larva em estádio diferente), col. SSMA-RS.

\section{Chave de diferenciação das pupas para as espécies de Thyrsopelma}

1. Pupa com mais de mais de 30 filamentos branquiais terminais (40-51)... T. itaunense (d'Andretta \& González,1964)

-. Pupa com menos que 30 filamentos branquiais terminais. 2.

2. Pupa com 20-24 filamentos branquiais terminais T. orbitale (Lutz,1910)

-. Pupa com menos que 20 filamentos branquiais terminais ... 3 .

3. Pupa com 15 filamentos branquiais terminais T. jeteri sp.n.

-. Pupa com 12 filamentos branquiais terminais.. 4.

4. Fronto-clípeo e tórax cobertos por grande quantidade 
de setas espatuliformes, esclerotizadas

T. hirtipupa (Lutz,1910)

-. Fronto-clípeo e tórax sem setas espatuliformes . .5 .

5. Fronto-clípeo e tórax com grande número de tubérculos do tipo subtriangular.. T. scutistriata (Lutz,1909)

-. Fronto-clípeo e tórax com tubérculos do tipo arredondado. ...6.

6. Espículas do tegumento da parte distal dos filamentos branquiais terminais desenvolvidas.......T. perplexa (Shelley et al.,1989)

-. Espículas do tegumento da parte distal dos filamentos branquais terminais pouco desenvolvidas

T. guianense (Wise,1911).

Observações adicionais (22.09.2005): Na semana anterior ao recebimento deste trabalho para serem efetuadas as correções sugeridas pelos consultores, saiu publicado um trabalho (Pepinelli,M. et. al. 2005), aonde é apresentada a descrição de uma nova espécie: Simulium (Thyrsopelma) duodenicornium Pepinelli; Hamada \& Trivinho-Strixino, aumentando então para oito o número de espécies para este gênero. T. jeteri sp.n. ao nível de imaturos, é nitidamente diferenciada desta espécie, pois T. duodenicornia apresenta (segundo a descrição original) as seguintes características:12 filamentos branquiais terminais; maior comprimento ventral (2,9-3,4 mm) / dorsal (4,0-4,4 mm); maior comprimento do último estádio larval $(6,2-7,5 \mathrm{~mm})$; maior número tanto de fileiras de ganchos (178-184) como de ganchos por fileira (2631) do disco anal; um maior número de divertículos por lóbulo anal (25-27); a proporção entre o I e o II segmento antenal da larva é muito maior (I:II = 1:2,5-2,6). A descrição de $T$. duodenicornia, amplia a variabilidade na relação dos segmentos antenais larvais para o gênero, passando a ser II $>$ III $\leqslant \geqslant I$.

\section{AGRADECIMENTOS}

Ao Dr. Assad Darwich, CPBA/INPA, pela análise das amostras de águas do arroio Marmeleiro. Ao Técnico Ulysses Carvalho Barbosa, CPCS/INPA, pelo auxílio no trabalho de campo. Ao Dr. Felipe Arley Pessoa (CPCS/LFV) e à Dra. Claudia Maria RíosVelásquez (CpM\&LD-FIOCRUZ/AM) por terem disponibilizado o seu tempo e o material microfotográfico existente na CpM\&LD-FIOCRUZ/AM com o qual foram obtidas as primeiras imagens digitais de $T$. jeteri.

\section{BIBLIOGRAFIA CITADA}

Bastos,R.L.T.; Hamada,N.; Luz,S.L.B. 2004. Citotaxonomia de Simulium hirtipupa Lutz (Diptera:Simuliidae) da Amazônia. In: XII Jornada de Iniciação Científica do PIBIC/CNPq/ FAPEAM/INPA: resumos expandidos, Manaus: FAPEAM/INPA, p. 165-166.

Coscarón, S. 1987. El gênero Simulium Latreille em la Región Neotropical: Análisis de los Grupos Supraespecíficos,
Espécies que los integran y Distribución Geográfica (Simuliidae,Diptera). Coleção Emilie Snethlage. Museu Paraense Emílio Goeldi. Belém, PA. 111pp.

Coscarón, S. 1991. Fauna de água dulce de la República Argentina. Insecta, Diptera, Simuliidae. Vol 38, fasc. 2. Conicet, Buenos Aires. 304 pp.

Crosskey, R.W. \& Howard, T.M. 2004. A revised taxonomic and geographical inventory of world blackflies (Diptera: Simuliidae). The Natural History Museum, 82pp. (versão digital, em pdf, com distribuição avulsa pelos autores) mas que pode ser obtida também em http://www.nhm.ac.uk/ research-curation/projects/blackflies/Inventory.pdf, $78 \mathrm{pp}$.

Enderlein, G. 1934 (1933). Weiterer Ausbaus des Systems der Simuliiden (Diptera). Deutsch. Ent. Zeitschr.: 273-292.

Hamada,N.; Ale-Rocha,R.; Luz, S.L.B. 2003. Description of Simulium damascenoi (Diptera: Simuliidae) Male and the Black-Fly Species from the State of Amapá, Brazil. Mem. Inst. Oswaldo Cruz, 98(3): 353-360.

Maia-Herzog,M.; Shelley,S.J.; Luna Dias,A.P.A. 1985. Simulium birtipupa Lutz, 1910 (Diptera:Simuliidae) - Descrição dos adultos e larva e redescrição da pupa. Mem. Inst. Oswaldo Cruz, 80(4): 483-490.

Miranda-Esquivel, D.R.; Coscarón,S. 2001. Cladistic analysis of Simulium (Trichodagmia) and Simulium (Thyrsopelma) (Diptera:Simuliidae). Zoological Journal of Linnean Society, 132: 429-439.

Shelley,A.J.; Lowry,C.A.; Maia-Herzog,M.; Luna Dias,A.P.A.; Moraes,M.A.P. 1997. Biosystematic studies on the Simuliidae (Diptera) of the Amazonia onchocerciasis focus. Bull. nat. Hist. Mus. Lond. (Ent.) 66 (1): 1-121.

Shelley,A.J.; Maia-Herzog,M.A.; Lowry, C.A.; Luna Dias,A.P.A.; Garritano, P.R.; Shelley,A.; Camargo,M.; Carter,H.G. 2000. The Simuliidae (Diptera) of the secondary onchocerciasis focus at Minaçu in central Brazil. Bull. nat. Hist. Lond. (Ent.) 69 (2): 171-221.

Pepinelli,M.; Hamada,N.; Trivinho-Strixino,S. 2005. Simulium (Thyrsopelma) uodenicornium, a new black fly species (Díptera: Simuliidae) from the outheast Region of Brazil. Zootaxa 1040: 17-29 (www.mapress.com/zootaxa/)

Py-Daniel,V. 1997. Oncocercose, uma endemia focal no hemisfério norte da Amazônia. In: Barbosa,R.I.; Ferreira,E.J.; Castellón,E.G. (Eds.) Homem, Ambiente e Ecologia no Estado de Roraima. Instituto Nacional de Pesquisas da Amazônia, Manaus, Amazonas. p.111-155

Py-Daniel,V.; Andreazze,R.; Medeiros,J.F. 2000. Projeto Piloto Xitei/Xidea (Roraima). I. Índices Epidemiológicos da Transmissão de Onchocerca volvulus (Leuckart,1893) para os anos de 1995-1996. Entomologia y Vectores, 7(4): 389444.

RECEBIDO EM 31/03/2005 ACEITO EM 18/10/2005 